\title{
A Qualitative Study on the Effects of Real-World Stimuli and Place Familiarity on Presence
}

\author{
Matti Pouke* \\ University of Oulu \\ VTT Technical Research Centre of Finland
}

Toni Alatalo ${ }^{\text {II }}$

University of Oulu

\author{
Johanna Ylipulli ${ }^{\dagger}$ \\ University of Helsinki \\ University of Oulu
}

\author{
Satu Rantala \\ University of Oulu
}

Timo Ojala"

University of Oulu

\author{
Paula Alavesa ${ }^{\S}$ \\ University of Oulu
}

\begin{abstract}
We present a qualitative study investigating the effects of real-world stimuli and place familiarity on presence. The study was carried out using a prototype Virtual Reality system designed for participatory urban planning. The system uses consumer grade VR hardware for viewing $3 \mathrm{D}$ virtual models of future architectural plans at an urban site. We collected comprehensive qualitative data from 16 participants who used the VR system on-site to immersively view and vote on three different future plans at the real-world location while exposed to ambient non-visual stimuli emanating from the physical location. We provide qualitative findings regarding presence along the four recurrent themes revealed by the thematic analysis of the research data: visual content, impact of physical surroundings, navigation and interaction, and suitability for participatory urban planning. We also analyse the findings in terms of feasibility of such on-site VR experience and the applicability of large-scale city models for VR.
\end{abstract}

Index Terms: H.5.1 [Information Interfaces and Presentation]: Multimedia Information Systems-Artificial, Augmented and Virtual Realities

\section{INTRODUCTION}

Urban planning often includes visual communication of plans and designs to the citizens that may be affected by the designs. In participatory urban planning the citizens are given an opportunity to provide feedback on the designs that may affect their final form. While the visual communication of plans is often done with maps, images and videos, also virtual 3D models and 3D environments, interactive and otherwise, have become commonplace. $[5,7,11,30$, 32]

New immersive technologies, such as Augmented Reality (AR) and Virtual Reality (VR) are attractive options for presenting urban designs leveraging 3D graphics. Handheld or wearable AR allows users to view designs in their actual physical context, for example, directly at the site of a planned new building. However, AR experiences can be limited in terms of their visual appeal (small field of view, rendering performance, poor visibility due to outdoor lighting), and their implementation at arbitrary locations can be difficult due to challenges in tracking and registration [28]. Virtual Reality (VR) experiences have little tracking problems with modern hardware,

*e-mail: matti.pouke@oulu.fi

†e-mail:johanna.ylipulli@helsinki.fi

¥e-mail:saru.rantala@oulu.fi

§e-mail:paula.alavesa@oulu.fi

Ie-mail:toni.alatalo@oulu.fi

"e-mail:timo.ojala@oulu.fi are rich in visual detail and can create strong, believable experiences $[19,26]$. They are, however, usually built to replace real-world stimuli instead of complementing it.

In the study described in this paper, we conduct an experiment in mixing virtual and real-world stimuli in a VR experience. We used consumer grade VR equipment so that futuristic architectural plans were presented for participants as an immersive VR experience while they were physically located at the actual real-world design site. The contribution of this study is to investigate the effect of real-world non-visual stimuli, as well as the familiarity of a place, on presence. In a similar vein to [6], we use qualitative methods to study breaks in presence (BIP). We analyze how real-world stimuli and place familiarity either enhances presence causes BIPs, or prevents the onset of presence. In addition, we study the general applicability of on-site immersive viewing of large-scale 3D virtual city models for urban participatory planning and serious VR applications. We consider our study topical, due to the increasing availability of consumer-grade mobile VR devices, such as GearVR, Oculus Go and Oculus Quest that could be used for similar purposes in the future.

\section{Related Research}

\subsection{Presence and Plausibility in Virtual Reality}

The most attractive feature of VR is its capability to produce deeply engaging experiences. One of the earliest key contributions in this scope is the work of Slater and Wilbur [26] that defined the concepts of immersion and presence. According to them, immersion refers to the objective technical capabilities of a system to produce rich sensory experiences while presence (also dubbed as place illusion) refers to the subjective sensation of being in a place. Essentially, presence is the extent of artificial sensory stimuli succeeding in replacing real ones [22]. High levels of presence are seen as especially beneficial for VR applications on therapy [19] and training [4], for example. Presence seems to have a circular relationship to emotion: VR applications eliciting emotions are experienced as ones causing high sense of presence, while high presence in turn helps to induce emotions [17].

Schloerb et al. [20] defined objective presence as a separate entity from subjective presence, as the user's ability to perform tasks within the remote environment, either virtual or a remote physical system through teleoperation. In his later work, Slater [23] introduced the concept of plausibility, which refers to the extent of an immersive system producing realistic responses in regards to user, as well as the overall credibility of the virtual scenario taking place.

Different methods for measuring and quantifying presence have been proposed. Slater and Wilbur [26] offered observing whenever the user is producing physical actions signifying his/her engagement in VR, for example, using unnecessary physical movements for dodging virtual objects. Several questionnaires offer subjective methods for quantifying presence, for example [25] and [31]. However, the measurement of presence is not straightforward: Usoh et al. [27] observed that these presence questionnaires failed in distin- 
guishing VR from true reality when the same place was depicted in both. Their conclusion was that it might not make sense to compare presence scores across different systems. Following up, Slater [22] further criticized the use of questionnaires as a sole method to study presence: it is unclear whether presence questionnaires can measure presence as a phenomenon that alters actual mental activity or behavior, or if they simply plant the idea of presence into the heads of the participants. Later, Slater [23] suggested treating presence simply as a binary variable; either the place illusion occurs, or it does not.

One way to measure presence is to study whether the VR application produces a similar behavioral or physiological response as a corresponding real-life situation would. For example, biometric measurements have been shown to be useful in detecting presence in VR applications simulating a stressful or a fear-inducing situation [13]. An obvious problem arises if the same approach is used to measure presence from a mundane situation. Slater et al. [24], however, found biometric measurements successful for detecting BIPs, as well as aspects related to the application content, such as speaking avatars in a bar setting not specifically designed to be stressful.

Schloerb et al. [20] suggested a quantitative measure of presence through psychophysiological approach. While technically appropriate for mirror-world studies, such as the one presented in the paper, the approach essentially requires the participants to find at least some isolated aspects of the VR system to be completely indistinguishable from true reality. Unfortunately as of now, it is extremely rare, if not impossible, to find systems with such a high degree of immersion.

An example of measuring plausibility can be seen in the work of Bergstrom et al. [3] where users were immersed in a virtual string quartet performance. The aim was not to produce an overall 'plausibility score', but instead to focus on identifying the most relevant application features affecting the subjects' sense of plausibility.

The approach of Garau et al. [6] to measure presence is perhaps closest to our study. They qualitatively evaluated presence in an experiment, where strong BIPs were deliberately caused by several whiteouts eliminating visual and audio cues. According to their findings, the participants experienced the whiteouts as strong sensations, such as 'startling' or 'waking up'. In addition, it was confirmed that presence can fluctuate temporally or even spatially. Participants reported presence as constantly decreasing, increasing, peaking or staying constant throughout the experiment, while the analysis also revealed that different parts of the VE (Virtual Environment) caused fluctuations in presence.

While our research method is qualitative as well, the main difference to Garau et al. [6] is that we do not cause deliberate BIPs. Instead, we investigate how live real-world stimuli and place familiarity either enhances presence, causes BIPs or prevents the onset of presence altogether. In addition, our experiment takes place in a setting where the participants comment on the urban plans of a design site in a VE while they are physically situated at the real-world location of the design site. Moreover, our prototype VE is rather large in comparison.

\subsection{D Models in the Context of Real-World Locations}

Many studies have confirmed the usefulness of 3D models in engaging the public in urban planning processes, technologies ranging from CGI-enhanced photomontages to VR (e.g. [7, 12,30]). Howard et al. [7] emphasized the usefulness of interactive VR in participatory urban planning; traditional public consultation often lacks in immersion, as well as interaction and feedback capabilities. According to their study, these shortcomings can be overcome by utilizing VR technology that allows users to interactively navigate the planning sites. However, it has also been stated that the usefulness of various 3D techniques in participatory urban planning is highly contextdependent, and a single superior approach does not exist [11].

Some studies have considered real-world urban locations in con-

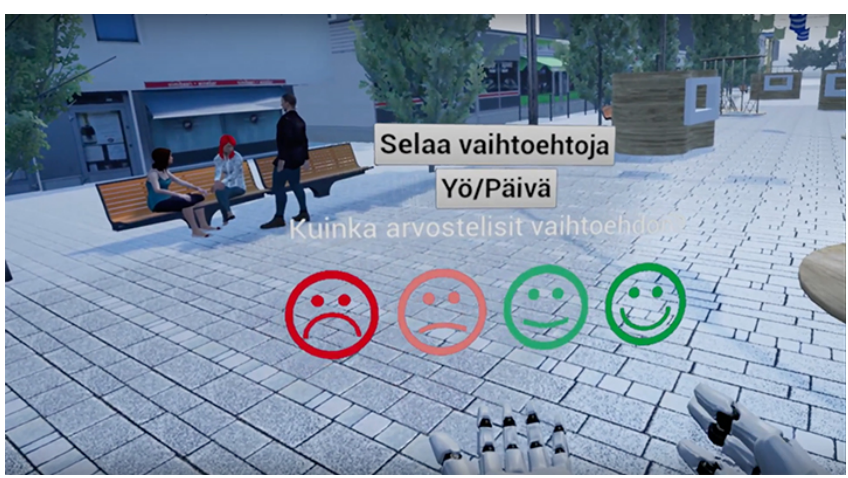

Figure 1: A screenshot of the UI. An upwards left palm invokes the UI while the right hand is used for pointing and selection.

junction with their VR counterparts. For example, Luigi et al. [12] studied the validity of a VR system in depicting real-world locations in participatory urban planning. They found that acoustically rich VR environments and real-world locations were perceived almost identical in terms of descriptive properties, such as pleasantness, calmness and vivaciousness. Alavesa et al. [2] used 3D city models as mirror worlds of corresponding real-world urban locations in a pervasive game, studying the effects of moving between the virtual mirror world and the physical real-world on co-presence and memorability during gameplay. Pouke et al. [16] used 3D interior models as mirror world in a VR interface for a public library.

Outside urban context, Jung et al. [8] investigated the effect of visual transition from real world into a similar VE on presence and body ownership. They found out that gradual transition was seen more beneficial for presence in comparison to an instantaneous transition. Simeone et al. [21] have conducted multiple studies on the interaction between real and virtual worlds, for example using virtual elements to affect user movement in the real world.

\section{Prototype VR System}

We developed an interactive prototype VR system for visualizing future architectural plans in the City of Oulu, Finland (population of roughly 200 '000 people). The system was developed as an Unreal Engine 4 application with Oculus Rift CV1 and Touch controllers as VR hardware. UE4 was chosen for its VR hardware support as well as more aesthetic lighting and material system that were seen beneficial for the development of an immersive VR application.

The 3D virtual models used in the application are based on an existing game engine based model depicting roughly 30 blocks of buildings, streets and vegetation of the City [1]. While it is somewhat uncommon to use game-engine models in the context of urban planning (more common approach is to convert GIS and CAD models into interactive 3D models [7]), our game engine model does correspond to reality. The coordinate system of the model is defined so that scene units correspond to the metric system and the axes are oriented among cardinal directions. The building models were originally placed using the city plan as a reference. The buildings are also rotated according to the actual geo-orientation of their realworld counterparts.

When the assets of the original city model were imported to Unreal Engine, some changes were made to the content. The original street meshes were deleted and replaced with a terrain system. Locations close to the design site had a "facelift". The street level detailing of the 3D buildings was improved. New content, such as human avatars and street furniture were placed in the scene close to the design site. In addition, large parts of the original materials were updated to replace plain diffuse textures with PBR materials [9].

The application utilizes the default Virtual Reality template of 
Unreal Engine 4 for navigation, interaction and visualization. Navigation uses teleportation instead of continuous movement since it has been shown to be an effective countermeasure against vectionbased cybersickness [10]. The VR system was configured so that navigation in the virtual scene would commence from a particular location, where we planned to place the VR equipment for data collection. However, the user was able to navigate throughout the whole virtual city model of about 30 blocks by using short-range teleportation.

The UI (Fig. 1) allows the user to shuffle through different architectural plans, as well as cast a vote indicating a preference of the plans. The 4-scale voting scheme and the graphical design of its 2D icons were adopted from a commercial voting application used by the City in other citizen participation activities. The user can switch between day and night mode in the scene. The UI was developed as an Unreal Engine widget. It is invoked by turning the palm of the left virtual hand skywards. The user can then select icons by pointing at them with the right virtual hand and squeezing a controller trigger button. The VR system had no audio, as we wanted the user to be able to hear the ambient sounds of the physical environment.

\section{Experimental Setting}

Initially, we created 3D virtual models for four sites around the city from the original 2D plans depicting alternative architectural designs for the sites in year 2040. In this paper, however, we focus on the data gathered from the most important site: a pedestrian street regarded as the "center" of the City and a very important landmark to many people. Currently, the pedestrian street does not have any cover. The future architectural plans depict three alternative ways of (partly) covering the pedestrian street (Fig. 2). Although the pedestrian street has minimal car traffic, it is still quite a noisy place.

\subsection{Data Collection and Analysis}

Data collection was conducted as follows. We set up a PC with VR equipment at the real-world location so that study participants were able to view future plans on-site using our VR system (Fig. 3). The experiment included participants that were recruited beforehand, as well as passersby that were recruited on the spot "in-the-wild" fashion [18]. Since the original architectural plans are public, they were familiar to some participants, however, showing them was not part of the study setup. Because the experiment took place on a pedestrian street, the participants were seated for their own safety. Before each session, researchers helped the participants to familiarize themselves with the controllers and the UI.

In total, we had 16 adult participants from the general public, 11 males and 5 females. From these participants, 12 (8M, 4M) were passersby and $4(3 \mathrm{M}, 1 \mathrm{~F})$ interviewees. It is possible that the smaller amount of women reflects the sociocultural meanings attached to new technologies; especially in the country where this study took place, females sometimes find new technology less attractive than men. [15, 29].

After a participant concluded using the VR prototype ("VR session"), we gathered qualitative data with a thematic semi-structured interview along the following themes: Utilizing VR in participatory urban planning, Experiences of the City centre and participatory planning in general, Experiences and attitudes towards new technology, Interaction and Presence.

In presence studies it is common to ask participants whether they felt more like being in one place or another, e.g. the laboratory or the place depicted by the VR system $[6,20]$. However, this was not feasible in our experiment, since both the real location and the VE were the same. Instead, we asked about existing in two locations at the same time, e.g. "How did it feel like being in two places at the same time, in physical and digital cities? Were there conflicts between these two?" and "How do you experience these two places, do you have any ideas for interaction between the

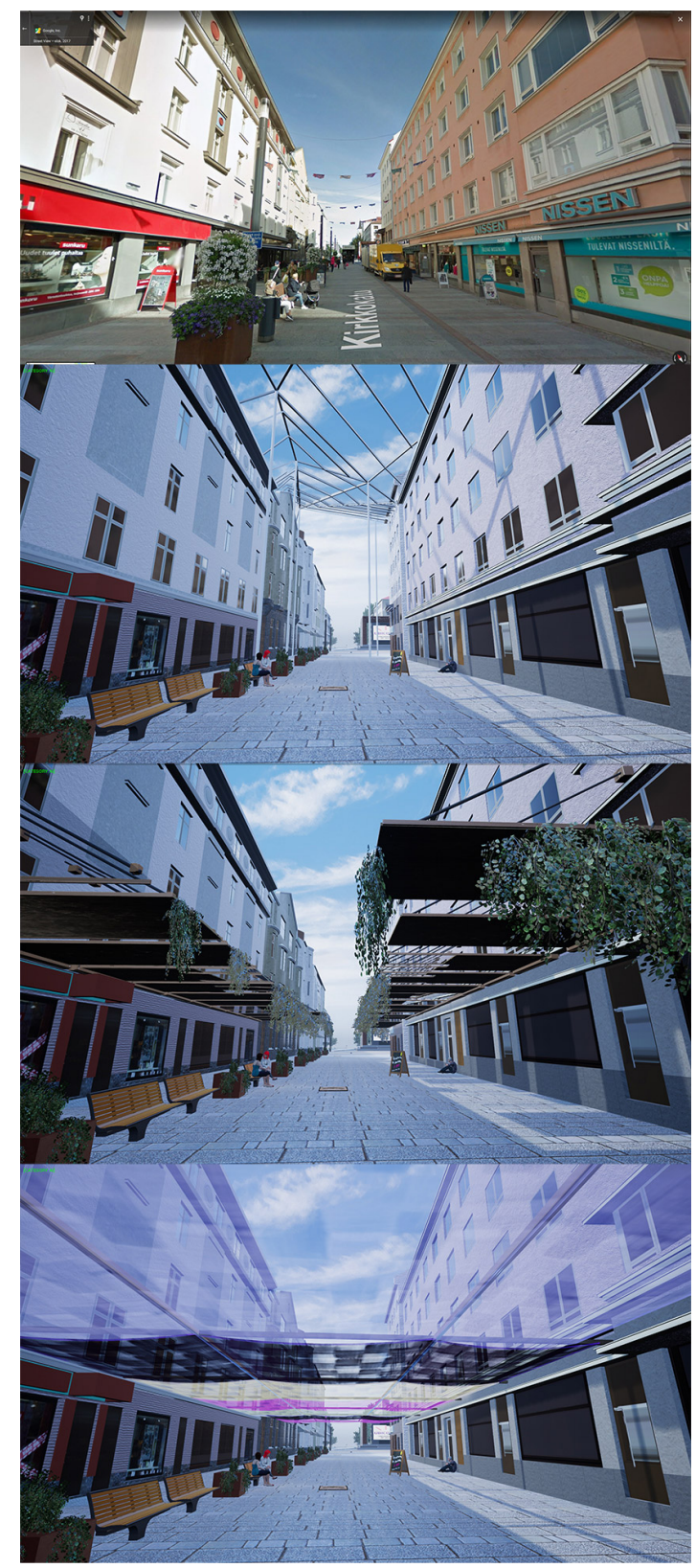

Figure 2: The experimental site - Real-world view (top) and virtual models of three alternative future plans of (partly) covering the street (below).

places?" Similar to Garau et al. [6], we occasionally used presence related questions from the Slater-Usoh-Steed questionnaire [25] in a qualitative manner, sometimes also asking for a rough quantification for reference. While the interviews themselves were conducted after each VR session, the participants did often speak aloud while using the prototype system. Neither the terms presence or BIPs were used in the interviews since we wanted to prevent participants from forming preconceived ides about the concepts of presence, or BIPs Instead, we interpreted the participants' comments and reactions to identify the aspects of the prototype that prevented the onset of presence (an overall property of the prototype felt artificial from the start), clearly caused BIPs (stating a particular property felt artificial), as well as occurrences when participants seemed clearly present (e.g. abundant body movements while using the system or qualitative 


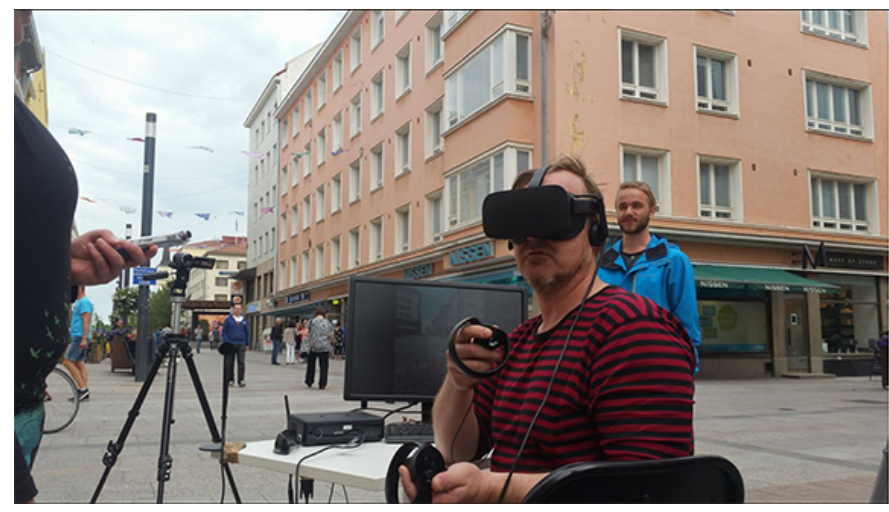

Figure 3: A study participant testing VR system on-site.

responses to the questions taken from the SUS questionnaire [25]).

All the collected materials were transcribed and the resulting texts were read and analyzed by several researchers to gather comprehensive impression of the participants' experiences. The research data was analyzed by using thematic analysis (e.g. [14]) that gave rise to four recurrent themes: visual content of the virtual scene, navigation and interaction, impact of physical surroundings, and suitability for participatory urban planning. We first report qualitative findings on presence along these five themes in Section 5. We will then discuss selected phenomena in Section 6.

\section{FINDINGS}

According to the data, the interviewees mostly experienced a strong sense of presence while the passersby had more mixed experiences. Some participants reported gradual overall transition in the level of presence. However, the transitions were more uniform in comparison to results by Garau et al. [6] The only comments made by the participants concerned the overall increase of presence during the VR session, e.g. "At the end I was like... when I take off the glasses, does it feel like I had dropped back here from outer space? In that way, it started to feel like... you know, weird".

\subsection{Visual content of the virtual environments}

Most of the participants somewhat enjoyed the visual content of the VE, however, the participants often complained that the pedestrian street was too empty and scarce in detail. While the real-world site is a busy location, the virtual environment was considered too desolate to represent the actual location. We argue that this was especially amplified because of place familiarity: the participants had a strong mental image of the regular day-to-day atmosphere of the pedestrian street. Two participants experienced BIPs due to the lack of visible body in the VE. Some participants also complained about the "grainy" or "game-like" visual appearance of the VE. Some participants mentioned, however, that the experience was very realistic despite aesthetic limitations.

\subsection{Navigation and interaction}

Initial difficulties or other complications with interaction sometimes prevented the onset of presence. Overall, however, navigation and interaction became fluent for most participants once they got used to the VR system. A few participants had minor difficulties at the beginning as they felt particularly nervous about the experiment. One of the participants had motor difficulties and needed assistance using the system. These difficulties in interaction caused mixed experiences of presence: the participants considered the overall experience to feel more virtual than real, but the VE felt more like a place instead of images. Voting for different design options was found easy, but some of the participants hoped for more options.
One of the participants mentioned that while navigating in the virtual pedestrian street, he forgot being seated in one place.

The participants clearly hoped that the VE would have contained other interactions in addition to voting. The desire to interact with the environment and talk with the virtual humans came up with most participants. BIPs, as well as the limits of plausibility, were often encountered as participants frequently searched for interactive objects but instead found the environment non-responsive. For example, one participant tried to sit on a virtual bench: "Yeah, if you were a ghost, would it feel like this?". The participants also mentioned the need for virtual characters typical for the real-world location, e.g. street fundraisers that would actively approach the user. BIPs caused by the lack of interaction by virtual avatars has been shown in previous studies (e.g. [3,6]).

One participant (passersby) attempted to constantly move around in the VE by physically moving instead of using the controllers, which can be seen as a strong indicator of presence. When asked the question "Please rate your sense of being in the virtual environment, on a scale of 1 to 7 , where 7 represents your normal experience of being in a place" from the SUS presence questionnaire [25], this participant responded with 7 . In addition, this participant felt the VE to be more like a place than images.

\subsection{Effect of physical surroundings on VR experience}

Some participants stated that the experience felt powerful because the experiment took place in the actual location. For example, the mere knowledge that the surrounding soundscape was not prerecorded made the experience feel significantly more realistic. A few participants were clearly startled when returning back to reality. One participant told that the slight mismatch between virtual and real locations felt "somehow, extremely crazy" when taking off the HMD "It was like I suddenly teleported from over there, back here".

BIPs also occurred due to contradictions between the VE and reality. For example, loud music prevented the onset of presence for one of the participants. In addition, the overcrowding of the actual location often came up as a contradictory auditory element that was clearly harmful for presence. The interviewees seemed to consider the surrounding soundscape more as a supporting element while some of the passersby considered it somewhat more distracting.

The starry sky of the night-time option was often admired, however, surprisingly many participants considered it inaccurate. Most of the participants did not clearly state how the night-sky was inaccurate, but one of them mentioned that the stars are usually not visible due to light pollution.

The familiarity of the place produced mixed reactions. In reality, people often visit the pedestrian street to spend leisure time and meet with other people. According to our analysis, this atmosphere did not transform into the VE due to its desolation and lack of interactive elements. On the contrary, some participants did state that the VE felt like an actual place due to the fact that they knew the place.

\subsection{Suitability for participatory urban planning}

In general, the participants considered the VR system to work well for representing future architectural plans and useful. The immersive experience of a plan changed a participant's perception: while the plan looked attractive in the original 2D image, it felt "claustrophobic" in the VE. This can be seen as evidence of the usefulness of presence in participatory urban design. The possibility to "see the designs at the location" as well as the ability to inspect them from multiple angles yielded some very positive comments. Some considered voting by choosing a smiley too simple. A recurrent theme in the interviews was related to changing seasons and weather. The participants thought that VR offers a great way to present urban plans during different seasons that can introduce many changes on visual appearance and experience of the urban environment (e.g. [33]). 


\section{Discussion}

\subsection{Presence}

The participants provided mixed reports on the onset of presence and frequency of BIPs. The interviewees seemed to experience a strong sense of presence while the passersby had more mixed experiences. The impact of physical surroundings on presence was mixed. Also place familiarity had both positive and negative impacts.

While most participants seemed to appreciate the aesthetics of the VE, often its visual appearance was considered sub-par or lacking in detail. Besides technical limitations, it can be speculated that using the application on-site could have had a negative effect on the perception of aesthetics. The participants had an instant direct comparison to the real-world view of the site that ruthlessly highlighted the limitations of its virtual counterpart. This issue could potentially be counteracted by utilizing the findings of Jung et al. [8], where gradual transition from real to virtual was seen beneficial in comparison to instant transfer. However, it should be noted that our participants did not feel that coarse or "game-like" graphics were specifically causing lack of presence. Instead, a general lack of detail and "emptiness" of the virtual pedestrian street was the factor that was reducing presence.

Occasionally the stimuli from true reality caused BIPs or even prevented the onset of presence. The background sounds of the real environment, especially loud ones, acted as a distraction as they typically created a stark contrast with what was taking place (or rather was not taking place) in the VE. This is in line with Slater's remark about multiple levels of immersion [23]: while a user can have a strong sense of visual illusion simultaneously with a completely different auditory stimulus, the place illusion will break down if the stimuli become contradictory. Some participants did remain under strong sense of presence even when discussing with researchers, however, the illusion broke down during contradicting audio stimulus (e.g. loud music). On the other hand, when background noises were not contradicting with the visual stimulus, the participants stated that the "real" soundscape strongly reinforced their presence.

The familiarity of the locations also resulted in a mixed response from the participants. While some participants stated that the familiarity of the virtual scene increased their presence, many participants also found that visual and/or behavioral discrepancies between the real and virtual locations had a negative effect.

It was also stated that the fact that users could not see their own body distracted presence. It is a known phenomenon in VR systems [23], and we believe it was related neither to real-world stimuli nor place familiarity.

As a side note, a research assistant involved in conducting data collection reported that the feeling of wind and sunlight on his skin added positively to his experience. However, such experiences were not reported by study participants.

\subsection{Large virtual city models and plausibility}

One of the prevalent findings was that the participants hoped for more interaction capabilities and "small details" in virtual environments. This was often also highlighted by the fact that the site was familiar to the users but did not otherwise act as expected (for example, lack of crowds). The participants constantly tried to interact with various elements in the VE which were often unresponsive. We consider this to be something that should be considered especially in using large 3D virtual city models for interactive applications: a large-scale city model offers endless possibilities for users to probe for the limits of interactivity and find discrepancies breaking plausibility. This is further emphasized if the user is familiar with the original environment and has expectations about its behavior. The users will expect lively places to be full of people also in the VE, not to mention that the virtual people should elicit realistic responses, as well.
Previous research by Slater et al. [23] suggests that a VE should be plausible in terms of its intended purpose in order to be effective. While our findings suggest that our VR system could be considered successful in terms of participatory urban planning, they also suggest that interactive 3D city models are not automatically "general purpose" virtual reality models suitable for all applications out of the box. While such city models can offer a starting point for various domains ranging from serious applications to entertainment, considerable effort might be needed to keep the large-scale environment plausible, depending on its purpose.

\subsection{Suitability for participatory urban planning}

The experiment elicited positive responses related to using VR for participatory urban planning. Regardless of the perceived lack in visual aesthetics, the participants found the VR system useful for participatory urban planning. This implies that the relevant aspects of the design site were communicated regardless of graphical fidelity. This is in line with the findings of Luigi et al. [12], where descriptive properties of the environments were almost identical between virtual and real world locations. According to our study participants, the plans were easier to understand in comparison to $2 \mathrm{D}$ images as it was possible to navigate within the designs and inspect them from various angles. Some participants felt that the VR was a more realistic representation of how the designs would actually look and feel in real life.

While some participants considered the VR system to be difficult to use, eventually most of them learned to use the system fluently during the relatively short timespan of their VR sessions.

\subsection{Limitations}

In this paper, the analysis was limited only on data collected from a single experimental site, the pedestrian street. The site we focused on did not suffer from vehicular traffic which was a favorable condition for mixing real and virtual data. While our findings were rather positive regarding the mixing of real and virtual stimuli, they might not be generalizable into more difficult settings.

The VR system had some limitations, particularly in the aesthetic quality of the VE. As stated earlier, they are based on an earlier game engine model [1] that was developed for a web-based platform containing only diffuse materials and otherwise few details in terms of street-level objects. The visual quality of the VE was updated in the vicinity of the design site. However, the participants often did not stay in the vicinity, but instead leisurely explored the whole city VE. The appearance of the non-updated areas might have contributed to the overall level of aesthetics experienced by the participants, leading to spatial fluctuations in presence as reported by Garau et al. [6]. Feedback regarding lack of small details and properties were apparent throughout the findings; even though details such as street furniture, pedestrians and vegetation was placed into the VE, the participants observed the VE to be too desolate.

Other technical aspects that might have had a negative impact on aesthetics include reliance on dynamic lighting and the display resolution of the VR headset. The existence of lightmaps and global lighting would most likely had a positive impact on aesthetics, but they were not used due to the limitations of the original city model.

\section{CONCLUSION}

We presented a prototype VR system for participatory urban planning. The virtual scenes used in the prototype were adapted from a game-engine based 3D virtual city model consisting of approximately 30 city blocks. We created $3 \mathrm{D}$ virtual models of candidate future architectural plans of a real-world site. The plans were viewed and voted on with a consumer grade VR hardware. We collected qualitative data from 16 study participants that used the VR system on-site at the true real-world location of the site. 
We observed that the ambient soundscape has the potential to greatly reinforce presence. On the other hand, if the soundscape becomes contradictory it may cause BIPs or even prevent the onset of presence. This confirmed that sounds are a powerful component of presence as well as plausibility [3,23] and strongly affect the perceived ambience of an urban space [12]; the sounds of the surrounding real environment should not contradict with the visual input of virtual environment [23]. In mirror world systems, such as the $3 \mathrm{D}$ virtual city model used in this study, designers should not only be well aware of the actual ambience of the environment, but also prepare for the unpredictability of the real world soundscape.

Further, familiarity increases expectations on the behavior of the place. We found that a large-scale city model can be especially challenging in terms of plausibility due to the necessity for an equally large reservoir of interactive content. Familiar-looking VEs do, however, have the potential to reinforce the sense of place, which in turn has a positive impact on presence.

In the future, we plan to extend our analysis into other sites besides the pedestrian street. In addition, we wish to deepen our analysis not only regarding presence, but through theories of place in social sciences as well.

\section{ACKNOWLEDGMENTS}

This work was supported by the COMBAT project (293389) funded by the Strategic Research Council at the Academy of Finland.

\section{REFERENCES}

[1] T. Alatalo, T. Koskela, M. Pouke, P. Alavesa, and T. Ojala. Virtualoulu: collaborative, immersive and extensible $3 \mathrm{~d}$ city model on the web. In Proceedings of the 21st International Conference on Web3D Technology, pp. 95-103. ACM, 2016.

[2] P. Alavesa, M. Pakanen, A. Voroshilov, H. Kukka, A. Samodelkin, T. Ojala, and M. Pouke. City knights: Spatial realism and memorability of virtual game scenes in pervasive gameplay. In 20179 th International Conference on Virtual Worlds and Games for Serious Applications (VS-Games), pp. 71-78, Sept 2017. doi: 10.1109/VS-GAMES.2017. 8055813

[3] I. Bergström, S. Azevedo, P. Papiotis, N. Saldanha, and M. Slater. The plausibility of a string quartet performance in virtual reality. IEEE transactions on visualization and computer graphics, 23(4):1352-1359, 2017.

[4] F. Biocca. The cyborg's dilemma: Progressive embodiment in virtual environments. Journal of computer-mediated communication, 3(2):JCMC324, 1997.

[5] D. Bouchlaghem, H. Shang, J. Whyte, and A. Ganah. Visualisation in architecture, engineering and construction (aec). Automation in construction, 14(3):287-295, 2005.

[6] M. Garau, D. Friedman, H. R. Widenfeld, A. Antley, A. Brogni, and M. Slater. Temporal and spatial variations in presence: Qualitative analysis of interviews from an experiment on breaks in presence. Presence, 17(3):293-309, June 2008. doi: 10.1162/pres. 17.3.293

[7] T. L. Howard and N. Gaborit. Using virtual environment technology to improve public participation in urban planning process. 133(4):233241.

[8] S. Jung, P. J. Wisniewski, and C. E. Hughes. In limbo: The effect of gradual visual transition between real and virtual on virtual body ownership illusion and presence. In Proceedings of the 2018 conference on Virtual Reality and 3D User Interfaces. IEEE, 2018.

[9] B. Karis and E. Games. Real shading in unreal engine 4. Proc. Physically Based Shading Theory Practice, pp. 621-635, 2013.

[10] G. Llorach, A. Evans, and J. Blat. Simulator sickness and presence using hmds: comparing use of a game controller and a position estimation system. In Proceedings of the 20th ACM Symposium on Virtual Reality Software and Technology, pp. 137-140. ACM, 2014.

[11] A. Lovett, K. Appleton, B. Warren-Kretzschmar, and C. Von Haaren. Using 3d visualization methods in landscape planning: An evaluation of options and practical issues. Landscape and Urban Planning, 142:85-94, 2015.
[12] M. Luigi, M. Massimiliano, P. Aniello, R. Gennaro, and P. R. Virginia. On the validity of immersive virtual reality as tool for multisensory evaluation of urban spaces. 78:471-476.

[13] M. Meehan, B. Insko, M. Whitton, and F. P. Brooks, Jr. Physiological measures of presence in stressful virtual environments. ACM Trans. Graph., 21(3):645-652, July 2002. doi: 10.1145/566654.566630

[14] M. Q. Patton. Qualitative research. Encyclopedia of statistics in behavioral science, 2005.

[15] O. PISA. Pisa: Results in focus. Organisation for Economic Cooperation and Development: OECD, 2015.

[16] M. Pouke, J. Ylipulli, I. Minyaev, M. Pakanen, P. Alavesa, T. Alatalo, and T. Ojala. Virtual library: Blending mirror and fantasy layers into a vr interface for a public library. In Proceedings of the 17th International Conference on Mobile and Ubiquitous Multimedia, pp. 227-231. ACM, 2018.

[17] G. Riva, F. Mantovani, C. S. Capideville, A. Preziosa, F. Morganti, D. Villani, A. Gaggioli, C. Botella, and M. Alcañiz. Affective interactions using virtual reality: the link between presence and emotions. CyberPsychology \& Behavior, 10(1):45-56, 2007.

[18] Y. Rogers. Interaction design gone wild: striving for wild theory. Interactions, 18(4):58-62, 2011.

[19] M. V. Sanchez-Vives and M. Slater. From presence to consciousness through virtual reality. Nature Reviews Neuroscience, 6(4):332, 2005

[20] D. W. Schloerb. A quantitative measure of telepresence. Presence: Teleoperators \& Virtual Environments, 4(1):64-80, 1995.

[21] A. L. Simeone, I. Mavridou, and W. Powell. Altering user movement behaviour in virtual environments. IEEE transactions on visualization and computer graphics, 23(4):1312-1321, 2017.

[22] M. Slater. How colorful was your day? why questionnaires cannot assess presence in virtual environments. Presence: Teleoperators \& Virtual Environments, 13(4):484-493, 2004.

[23] M. Slater. Place illusion and plausibility can lead to realistic behaviour in immersive virtual environments. Philosophical Transactions of the Royal Society B: Biological Sciences, 364(1535):3549-3557, 2009.

[24] M. Slater, C. Guger, G. Edlinger, R. Leeb, G. Pfurtscheller, A. Antley, M. Garau, A. Brogni, and D. Friedman. Analysis of physiological responses to a social situation in an immersive virtual environment. Presence: Teleoperators and Virtual Environments, 15(5):553-569, 2006.

[25] M. Slater, M. Usoh, and A. Steed. Depth of presence in virtual environments. Presence: Teleoperators \& Virtual Environments, 3(2):130-144, 1994.

[26] M. Slater and S. Wilbur. A framework for immersive virtual environments (five): Speculations on the role of presence in virtual environments. Presence: Teleoperators \& Virtual Environments, 6(6):603-616, 1997.

[27] M. Usoh, E. Catena, S. Arman, and M. Slater. Using presence questionnaires in reality. Presence: Teleoperators \& Virtual Environments, 9(5):497-503, 2000.

[28] D. Van Krevelen and R. Poelman. A survey of augmented reality technologies, applications and limitations. International journal of virtual reality, 9(2):1, 2010.

[29] M. Vehviläinen. Gender, expertise and information technology. University of Tampere, Department of Computer Science, 1997.

[30] K. Wanarat and T. Nuanwan. Using 3d visualisation to improve public participation in sustainable planning process: Experiences through the creation of koh mudsum plan, thailand. 91:679-690. doi: 10.1016/j. sbspro.2013.08.469

[31] B. G. Witmer and M. J. Singer. Measuring presence in virtual environments: A presence questionnaire. Presence, 7(3):225-240, 1998.

[32] H. Wu, Z. He, and J. Gong. A virtual globe-based 3d visualization and interactive framework for public participation in urban planning processes. Computers, Environment and Urban Systems, 34(4):291298, 2010.

[33] J. Ylipulli, A. Luusua, H. Kukka, and T. Ojala. Winter is coming: introducing climate sensitive urban computing. In Proceedings of the 2014 conference on Designing interactive systems, pp. 647-656. ACM, 2014. 